\title{
Levantamiento de información geoespacial con la aplicación Input para el manejo de residuos sólidos, Upala, Costa Rica
}

\author{
Geospatial data collection with the Input Application \\ for solid waste management, Upala, Costa Rica
}

\section{Luis Diego Mora-Jiménez*}

\author{
DOl: https://doi.org/10.22458/rb.v32i1.3547
}

Recibido- Received: 15/02/2021 / Corregido- Revised: 17/03/2021 / Aceptado- Accepted: 31/03/2021

\section{RESUMEN}

El levantamiento de información geoespacial con la aplicación Input, se realizó en los distritos de Canalete y Bijagua, específcamente en las comunidades de Santa Cecilia, Las Brisas, Las Milpas, Armenias, San Isidro de Aguas Claras, Río Negro, La Torre y Guayabal. Estas comunidades no cuentan con un servicio de recolección de residuos sólidos que les permita a sus pobladores una correcta gestión de sus residuos, no como sucede actualmente, pues deben quemarlos o enterrarlos. El objetivo principal fue determinar la cantidad de usuarios, ingresos y porcentaje de morosidad que permita la planificación en la ampliación de la cobertura del servicio de recolección de residuos sólidos, mediante la toma de datos geoespaciales utilizando el sistema de información geográfica QGIS y la aplicación para teléfono móviles Input, mediante los cuales se creó un formulario que permitió registrar la información en campo de una manera rápida y sincronizarla en una computadora que se encontraba en la Municipalidad de Upala.

Palabras clave: residuos sólidos; aplicaciones geoespaciales; sistemas de información geográfica; Input.

\section{ABSTRACT}

This article presents the results of a geospatial information survey that was carried out in the districts of Canalete and Bijagua, specifically in the communities of Santa Cecilia, Las Brisas, Las Milpas, Armenias, San Isidro de Aguas Claras, Río Negro, La Torre and Guayabal, using the Input application. Such communities do not have a solid waste collection service that allows residents to properly manage their waste, since, as it is currently the case, people must burn solid waste or bury it. The main objective of the survey was to determine the number of users, their income and defaulting percentage in order to set up an expansion plan for the solid waste collection service.By collecting geospatial data using the QGIS geographic information system and the Input mobile phone application, a form was created, and field information was quickly registered and synchronized to an available computerat the Upala City Hall.

Key words: solid waste; geospatial application; geographic information systems; Input.

* M.Sc. Universidad Estatal a Distancia, Costa Rica. Escuela de Ciencias Exactas y Naturales. Cátedra de Sociedad y Ambiente. Profesor tutor. Imoraj@uned.ac.cr

ID: https://orcid.org/0000-0001-8765-0919 


\section{Introducción}

La gestión de los residuos sólidos en Costa Rica es un reto para los cantones extensos en su área y en donde los centros poblacionales se encuentran distanciados, tal es el caso del cantón de Upala. Los datos indican una cobertura del servicio de recolección de residuos ordinarios y de valorizables de $30 \%$ de sus viviendas (3988 unidades cubiertas de 13294 identificadas en el año 2016). Lastimosamente, $70 \%$ de las viviendas queman o entierran los residuos. En la figura 1, se aprecian los distritos del cantón de Upala donde no se brinda ninguna alternativa para disponer adecuadamente los residuos sólidos. Por lo general, estos sitios son fronterizos o se encuentran alejados del centro del distrito primero de Upala, por tanto representa un gran reto para la Municipalidad de Upala lograr un $100 \%$ de cobertura en una extensión de $1581 \mathrm{~km}^{2}$. Si bien en el distrito de Aguas Claras $24 \%$ de la población expresa eliminar sus residuos por medio de camión recolector, lo cierto es que el servicio se brinda por un tercero mediante un camión de cajón y no por medio de camión recolector.

El servicio de recolección de residuos sólidos ordinarios en el cantón de Upala ha experimentado un aumento en las rutas desde el año 2016, específicamente cuando se realizó la ampliación hacia comunidades del distrito Upala como San Luis y Colonia Puntarenas y el distrito San José de Upala, pasando de una cobertura de $19 \%$ de unidades residenciales a $30 \%$ de cobertura. Sin embargo, el porcentaje sigue siendo bajo en momentos donde la cantidad de construcción de viviendas en los centros urbanos del cantón ha evidenciado un incremento (figura 2).

El crecimiento de la población en estos sitios, aunado a la demanda y la presión ejercida por el consumo de productos, las viviendas construidas sin el debido permiso y la falta de un mapa catastral actualizado, han generado el aumento de residuos sólidos que no cuentan con un tratamiento y disposición final adecuada, así como la morosidad por el no pago del servicio. Según el Estado de la

Figura 1

Porcentaje de viviendas que eliminan los RSU mediante distintos métodos

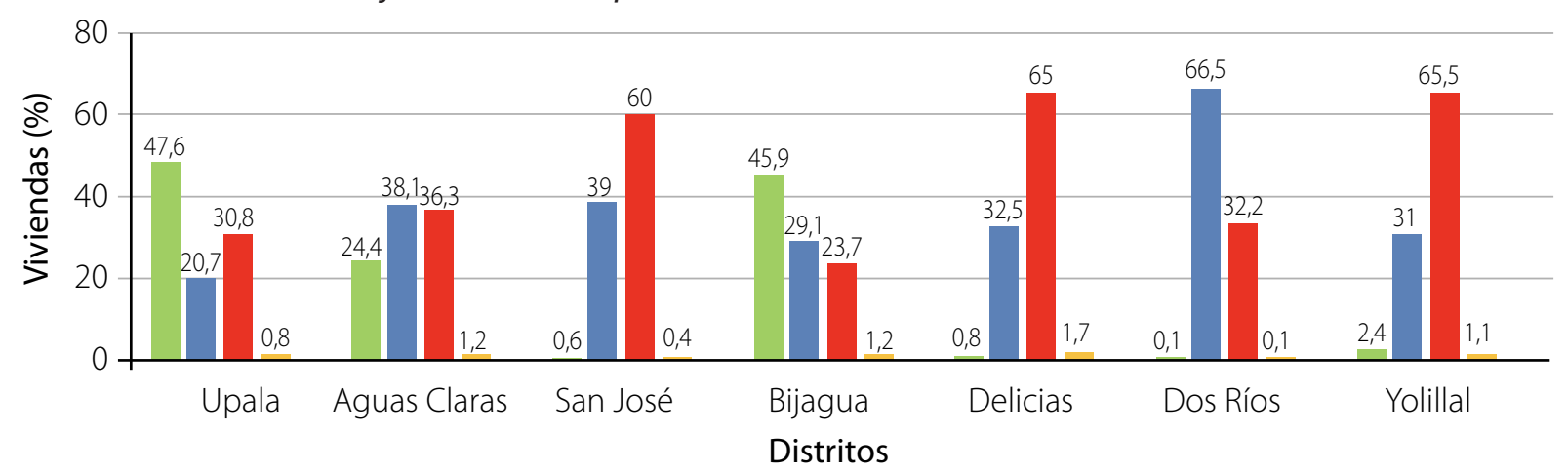

Porcentaje de viviendas que eliminan residuos sólidos por camión recolector

Porcentaje de viviendas que eliminan residuos sólidos botándolos

Porcentaje de viviendas que eliminan residuos sólidos quemándolos

Nota. La mayoría de las viviendas eliminan los residuos quemándolos. Elaboración propia con base en datos del Instituto Nacional de Estadística y Censo (2012). 
Figura 2

Permisos de construcción de vivienda 2016-2020, Upala

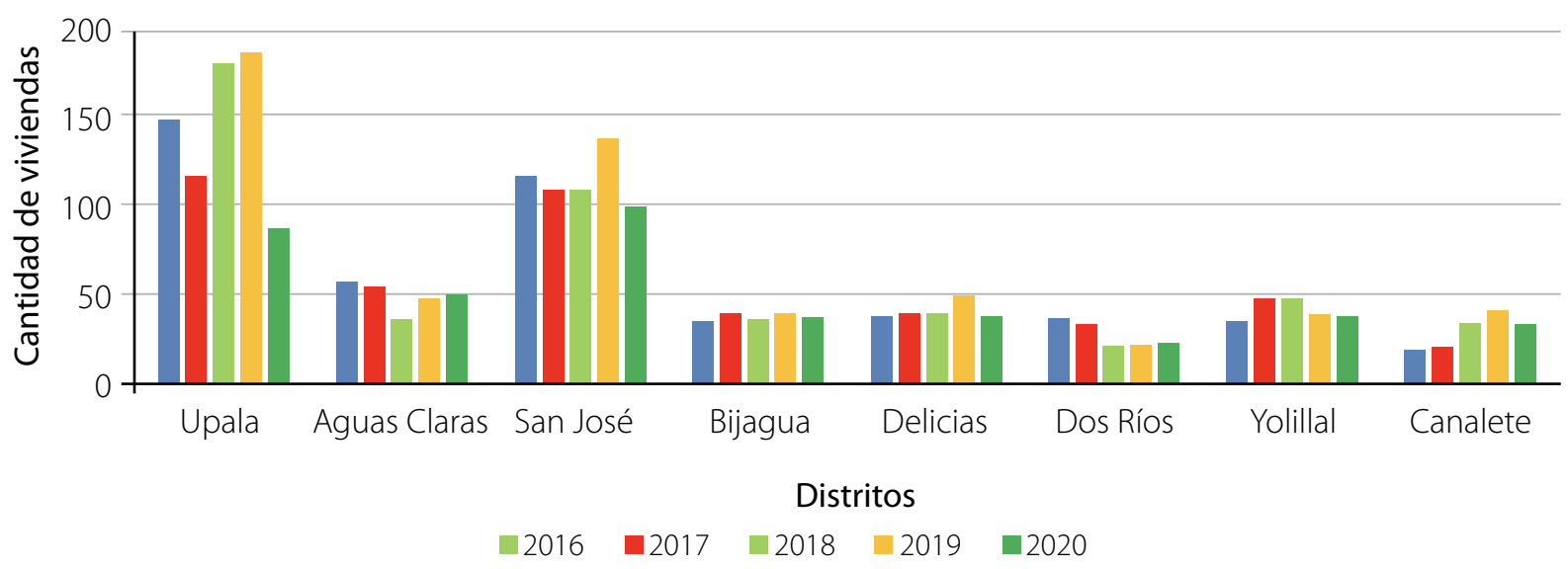

Nota. Elaboración propia con base en datos de la Municipalidad de Upala (2021).

Nación (2019), en su informe de Desarrollo Humano Sostenible, específicamente en la gestión de residuos sólidos en Costa Rica, los problemas asociados a la gestión integral de residuos sólidos son complejos debido a las elevadas cantidades y diversas calidades de los materiales desechados, el aumento en la población y el impacto de las nuevas tecnologías, con el consiguiente cambio en las materias primas, lo cual requiere una correcta planificación gubernamental y municipal.

Después del año 2016, la cantidad de permisos para vivienda ha aumentado gradualmente en distritos como Upala y San José de Upala, con un pico en el año 2019; no obstante, son distritos que tienen servicio de recolección. El tercer lugar, en cuanto a permisos de construcción de viviendas, es Aguas Claras y no posee servicio municipal de recolección de residuos sólidos.

Las poblaciones cercanas a las rutas de recolección en el distrito Upala, Canalete y Bijagua, son aprovechadas por los habitantes para disponer sus residuos en el área de cobertura del servicio sin ser usuarios, ocasionando gastos operativos sin el debido cobro.
También se observa la situación de la población en el distrito urbano de Aguas Claras y poblaciones cercanas como Armenias, Las Milpas, Santa Cecilia, donde sus pobladores deben enterrar o quemar los residuos generados, provocando una inadecuada gestión de los residuos $y$, a su vez, causan un detrimento de la salud ambiental y contaminación del suelo, los ríos, las quebradas y las calles.

Debido a esto, y como una forma de cumplir con el Artículo 8. Inciso d, de la Ley para la Gestión Integral de Residuos 8839, en la cual "las municipalidades deben garantizar que. en su territorio se provea del servicio de recolección de residuos en forma selectiva, accesible, periódica y eficiente para todos los habitantes [...]", fue necesario realizar un levantamiento geoespacial con la ayuda de una aplicación llamada Input desde un teléfono móvil. La utilización de este tipo de aplicaciones no es nueva, sin embargo, son un método tecnológico y moderno que facilita la recolección de datos en sitio y uso en diferentes campos de estudio, lo cual permite desarrollar distintas alternativas en el manejo y procesamiento de la información. 
Según Pacheco (2013), la tecnología celular y los dispositivos móviles han generado una masiva invasión de las infraestructuras de datos espaciales, las cuales facilitan el acceso a la información geográfica proveniente de diferentes fuentes (geoservidores), gracias a la disponibilidad de sensores (cámara, GPS, sonido, velocidad y movimiento) que permiten interactuar con el entorno.

De acuerdo con lo anterior, el proyecto pretende ser un insumo para la planificación del aumento de cobertura del servicio de recolección en comunidades del cantón y, a su vez, educar a la población beneficiada en el manejo de los residuos, los deberes y derechos dictaminados en el reglamento para la gestión integral de residuos sólidos en el cantón de Upala y evitar la morosidad que afecte la operación del futuro servicio.

\section{Materiales y métodos}

\section{Localización}

Los sitios de interés donde se pretende ampliar la cobertura de recolección de residuos sólidos se localizan en el distrito de Aguas Claras (San Isidro, La Torre, Río Negro y Guayabal) y Canalete (Santa Cecilia, Las Brisas, Las Milpas y Armenias), del cantón de Upala, los cuales son atravesados por la ruta nacional número 164. Además, son comunidades rurales asentadas desde la parte baja y media de las faldas del volcán Miravalles (figura 3).

Figura 3

Mapa de las comunidades del distrito de Canalete y Aguas Claras donde se ampliará el servicio de recolección de residuos sólidos ordinarios y valorizables

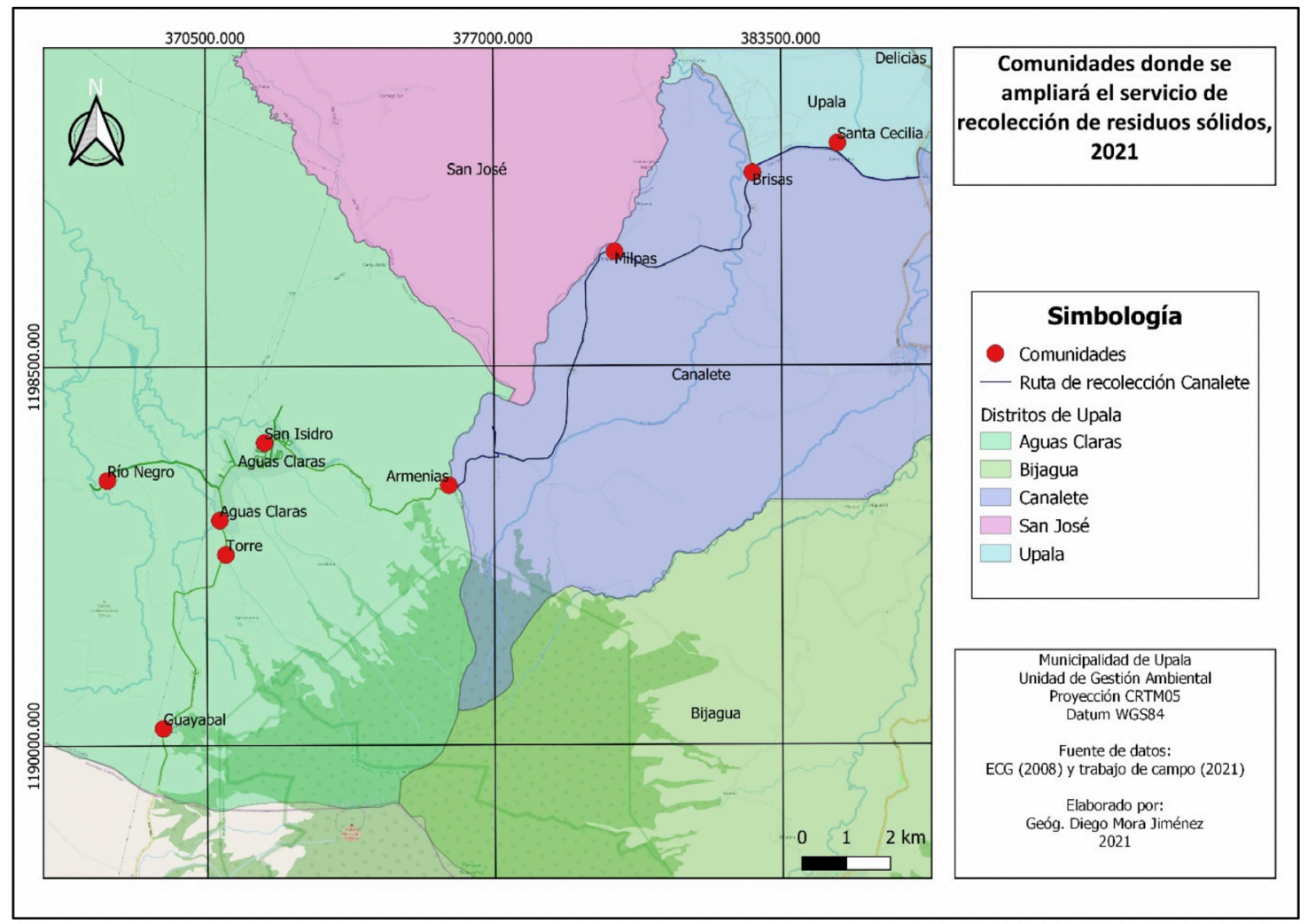

Nota. Elaboración propia con base en datos de la Escuela de Ciencias Geográficas (2006). 


\section{Campaña de información}

Un mes antes de iniciar con la preparación del proyecto y el levantamiento geoespacial de los usuarios, fue necesario realizar una campaña informativa en la red social de Facebook de la Municipalidad de Upala, con el objetivo de que las personas estuviesen preparadas con la información de su propiedad y de cómo consultarla desde sus teléfonos o computadora en el caso de no tenerla disponible.

\section{Preparación del proyecto}

La captura geoespacial de las viviendas y comercios en los sitios señalados se llevó a cabo por medio de una app llamada Input (gratuita y de código abierto), la cual trabaja basada en el sistema de información geográfica libre QGIS, lo que significa que se pueden crear proyectos desde el escritorio, para posteriormente capturar puntos, líneas, polígonos, y agregar información de interés mediante la realización de formularios personalizados; así mismo, añadir imágenes, videos y audios a la captura realizada (figura 4). Además, utiliza tecnología incorporada para el trabajo colaborativo por medio de un espacio que permite almacenar y sincronizar proyectos entre el escritorio y los teléfonos móviles, denominado Mergin (Lutra consulting, 2019).

En este caso, para el levantamiento geoespacial de viviendas y comercios, se creó un proyecto en el sistema de información geográfico QGIS de la zona de interés, con el fin de levantar información vectorial por medio de puntos y crear un formulario personalizado de atributos.

La información consultada a cada vivienda y comercio fue la siguiente: nombre del propietario, cédula del propietario, ubicación de la propiedad, correo electrónico del

Figura 4

A la izquierda se observa la página de la nube de datos Mergin y a la derecha la aplicación Input para teléfonos móviles

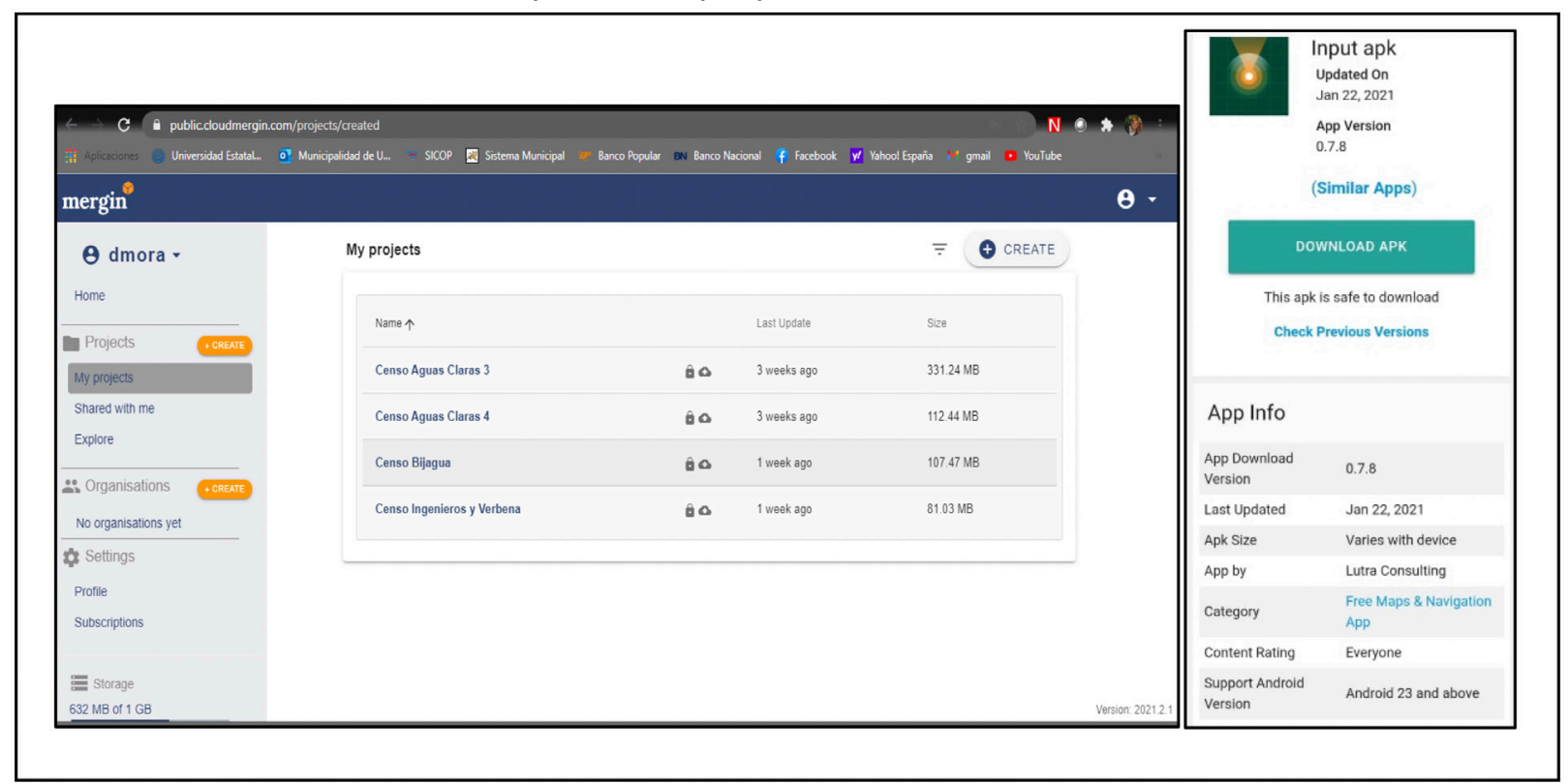

Nota. Elaboración propia con base en datos de Lutra consulting (2019). 
Figura 5

Aplicación del formulario personalizado

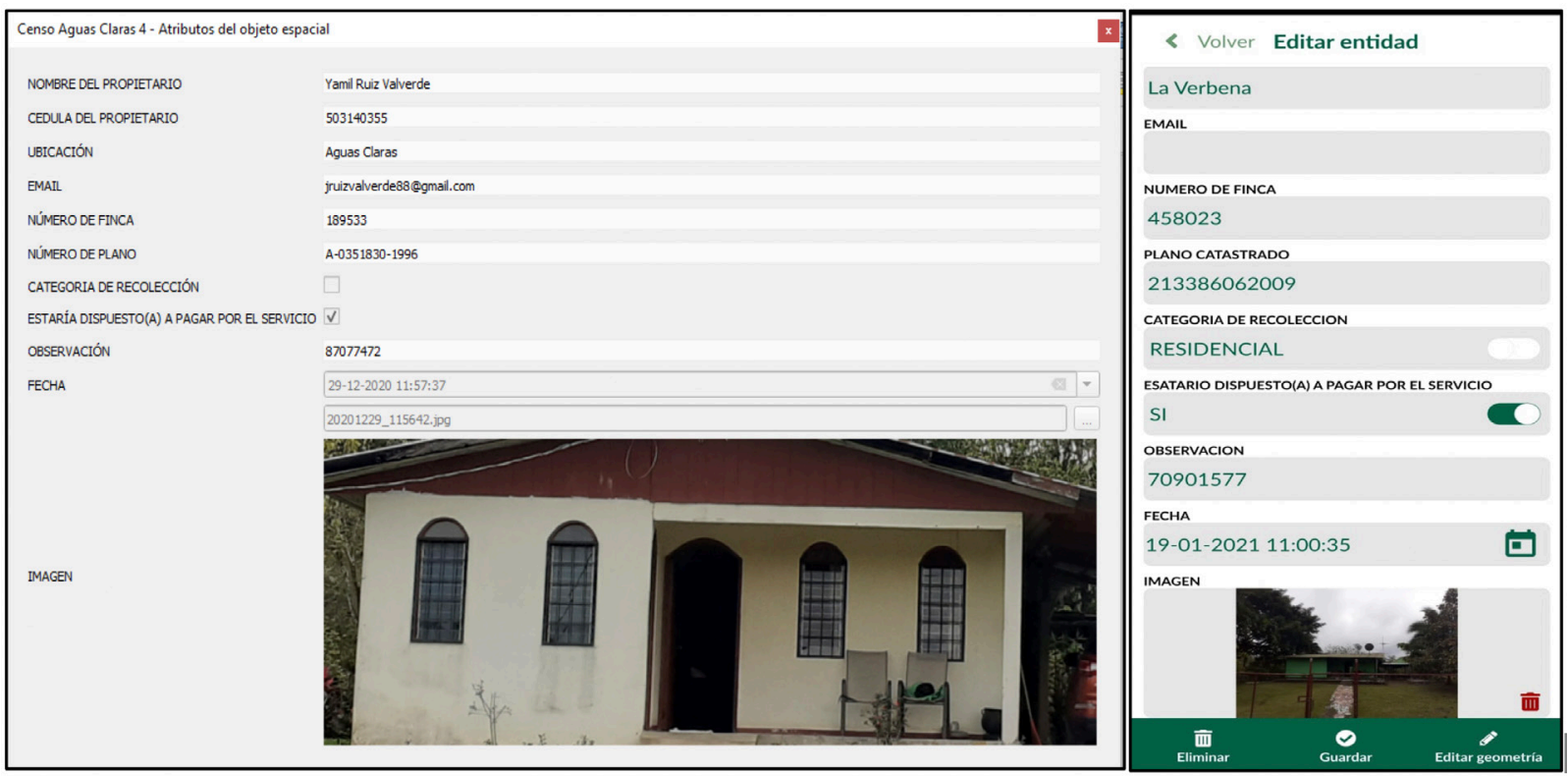

Nota. A la izquierda el formulario personalizado para el levantamiento de información geoespacial de los potenciales usuarios del servicio de recolección en el sistema de información geográfico QGIS a la derecha el formulario desplegado en la aplicación Input. Elaboración propia con base en software QGIS y aplicación Input (2021).

propietario, número de finca, número de plano catastrado, categoría del servicio de recolección (residencial o comercial), anuencia de pagar la tarifa de recolección, la fecha y hora del levantamiento y la imagen de la casa de habitación o comercio (figura 5).

Los datos del propietario, el número de finca (folio real) y la categoría de recolección sirven para registrar el usuario en el sistema municipal de contribuyentes; mientras que la pregunta sobre si está dispuesto o dispuesta a pagar por el servicio de recolección, aporta el porcentaje de aprobación para asegurar una morosidad baja en el servicio. Por último, la fecha y hora del levantamiento geoespacial y la imagen de la propiedad se utiliza como control de calidad de la información, así como para facilitar la búsqueda de información o realizar una segunda visita, si fuese necesario.

\section{Trabajo de campo}

Posteriormente, el proyecto se sincronizó desde el QGIS mediante el complemento de Mergin, para ser nuevamente sincronizado desde el teléfono móvil inteligente, donde se captura el punto del potencial usuario del servicio (ubicación geográfica) y los datos del formulario sin necesidad de tener una red de datos móviles (figura 6).

Una vez la información fue recaba en campo, se pudo sincronizar desde cualquier punto del sitio de interés, donde el censista se encontraba, hacia el escritorio del proyecto en la Municipalidad de Upala, lo cual permitía trabajar en la base de datos de forma simultánea, por ejemplo, corroborando la información del usuario con el sistema municipal de contribuyentes o por medio de una consulta desde la página web del Registro Nacional. 
Figura 6

Trabajo de campo

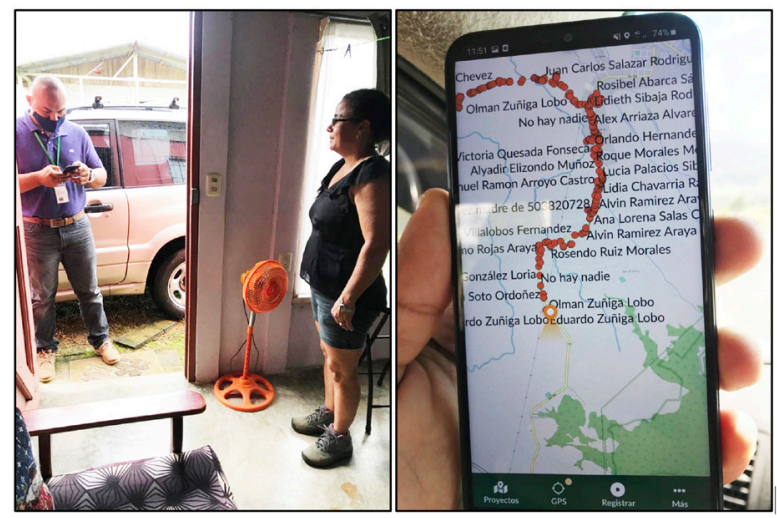

Nota. A la izquierda, el censista tomando los datos del posible usuario del servicio de recolección en Las Milpas desde el teléfono móvil con la aplicación Input y a la derecha los puntos censados desde la aplicación Input en el teléfono móvil. Fotografías de Mora, 2021.

\section{Resultados}

El levantamiento de la información geoespacial permitió registrar a un total de 1202 usuarios, de los cuales en 91 viviendas no había ninguna persona y 16 viviendas se encontraban en estado de abandono, para un total efectivo de 1095 usuarios que se les brindará el servicio de recolección de residuos sólidos. Las dos comunidades con la mayor cantidad de usuarios registrados fueron Aguas Claras y Las Milpas con un total de 655 usuarios y 114 usuarios respectivamente (figura 7).

La mayoría de las personas que se beneficiarían del servicio de recolección habitan en el distrito de Aguas Claras, específicamente en su centro llamado de la misma manera.

Por otro lado, de la cantidad de usuarios registrados, 1101 son unidades habitacionales, 78 tienen una actividad comercial y siete son instituciones del Estado. Lo anterior,implica un incremento de $8 \%$ de la cobertura actual en el cantón de Upala del servicio de recolección, el cual pasaría de 30\% a 38\% (5089 unidades que serían cubiertas de 13294$)$. La mayor cantidad de unidades habitacionales, en comparación con otras categorías, también permite interpretar que la generación de residuos sólidos será menor que en otros distritos donde la cantidad de comercios eleva las toneladas recolectadas al día como sucede en los distritos de Upala y Bijagua.

El levantamiento también permitió trazar la posible ruta del camión recolector de residuos sólidos ordinarios y valorizables, con una extensión aproximada de $50 \mathrm{~km}$, en su

Figura 7

Usuarios registrados del servicio de recolección de residuos sólidos por comunidades

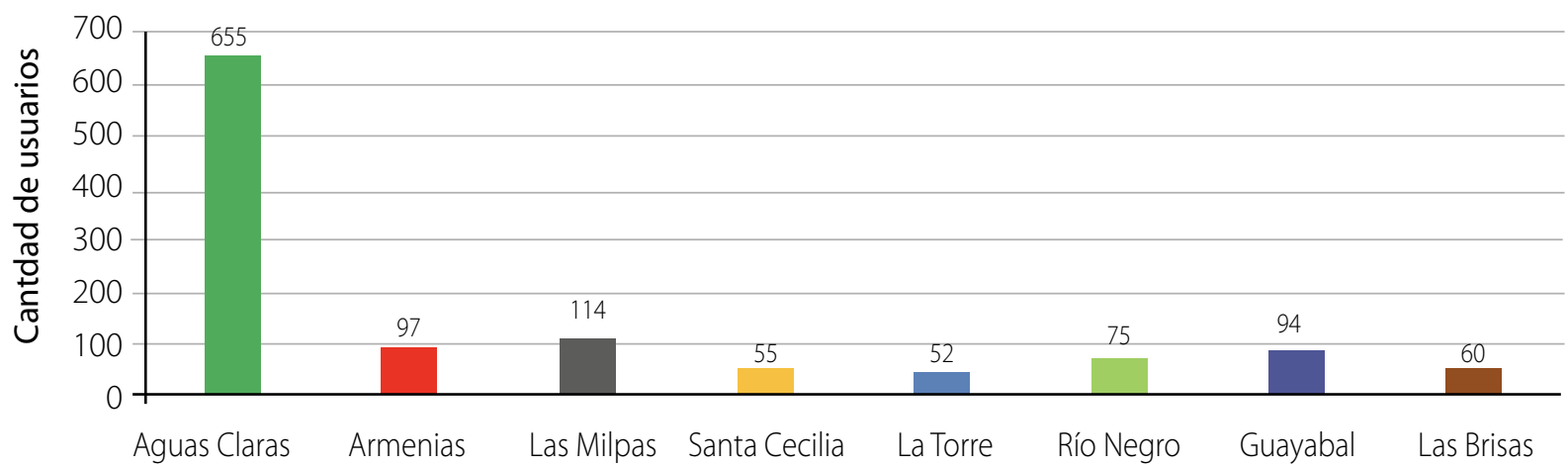

Comunidades

Nota. Elaboración propia con base en datos de la Municipalidad de Upala (2021). 
mayoría en camino de lastre. Si lo comparamos con otras rutas de recolección que realiza la Municipalidad de Upala, la cantidad de kilómetros posibilita que la ruta se pueda realizar en un tiempo aproximado entre 6 a 7 horas, lo cual fue determinado en el estudio de optimización de las rutas de recolección, realizado en el año 2019 (figura 8).

Otro resultado de importancia fue determinar la potencial recaudación de dinero por el cobro de las tasas por categoría del servicio de recolección de residuos, de acuerdo con la última actualización tarifaria del año 2020 (tabla 1).
Tabla 1

Categoría del servicio de recolección de residuos sólidos y sus tarifas

\begin{tabular}{|c|c|}
\hline Categoría & Tarifa \\
\hline Residencial & $\$ 3535$ \\
\hline Institucional 1 & \7075 \\
\hline Institucional 2 & $\$ 70740$ \\
\hline Comercial 1 & $\$ 8840$ \\
\hline Comercial 2 & $\$ 17685$ \\
\hline Comercial 3 & $\$ 26525$ \\
\hline Comercial 4 & $\$ 42445$ \\
\hline
\end{tabular}

Nota. Elaboración propia con base en datos de la Municipalidad de Upala (2021).

\section{Figura 8}

Mapa de puntos del levantamiento geoespacial con la aplicación INPUT y las posibles rutas de recolección de residuos sólidos en el distrito Canalete y Aguas Claras
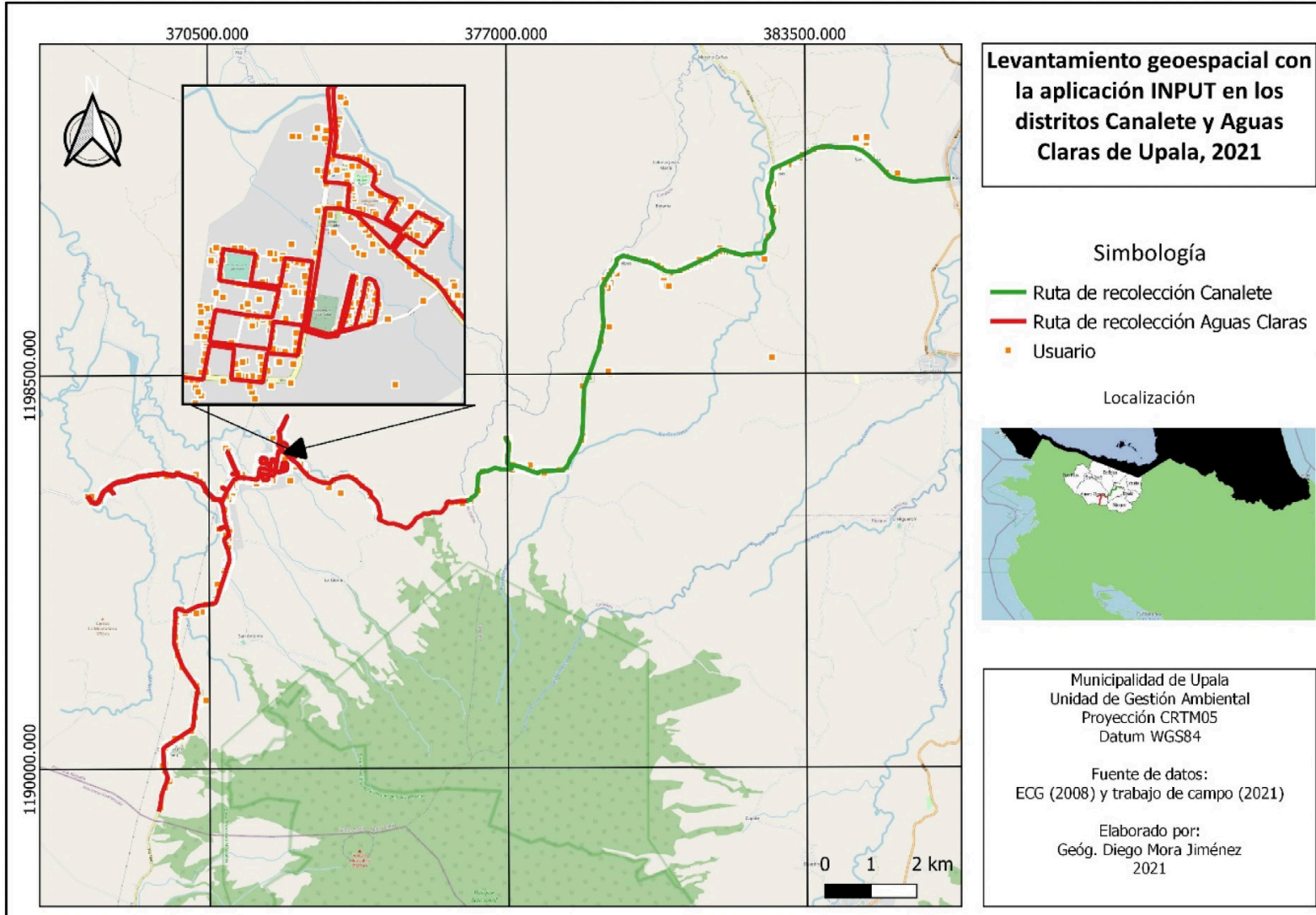

Nota. Elaboración propia con base en datos de la Escuela de Ciencias Geográficas (2006). 
Tabla 2

Ingresos de la ampliación del servicio de recolección de residuos sólidos

en las rutas Canalete y Aguas Claras

\begin{tabular}{c|c|c|c|}
\hline Categoría & Cantidad de usuarios & Ingreso mensual & Ingreso anual \\
\hline Residencial & 1101 & $\$ 3892035,00$ & $\$ 46704420,00$ \\
\hline Comercial 1 & 56 & $\$ 495040,00$ & $\$ 5940480,00$ \\
\hline Comercial 2 & 17 & $\$ 300645,00$ & $\$ 3607740,00$ \\
\hline Comercial 3 & 4 & $\$ 106100,00$ & $\$ 1273200,00$ \\
\hline Institucional 1 & 7 & $\$ 49525,00$ & $\$ 594300,00$ \\
\hline Total & 1185 & $\zeta 4843345,00$ & $\$ 58120140,00$ \\
\hline
\end{tabular}

Nota. Elaboración propia con base en datos de la Municipalidad de Upala (2021).

En la tabla 2, se muestran los ingresos mensuales y anuales por categoría por la futura ampliación del servicio de recolección de residuos sólidos ordinarios y valorizables en las comunidades de los distritos de Aguas Claras y Canalete, tomando en cuenta la tabla 1 para el cálculo.

El monto mensual por recaudar sería de ¿4843345 y el monto anual de $\$ 58120140$, de los cuales se tendrán que restar los costos directos, indirectos y de inversión del servicio de recolección, con el fin de determinar la factibilidad económica de la ampliación.

Otro dato relevante fue que, del total de usuarios registrados, 1075 respondieron a la siguiente consulta: ¿estaría dispuesto o dispuesta a pagar por el servicio de recolección de residuos sólidos? Como resultado 819 usuarios respondieron sí y 256 usuarios respondieron no; es decir, $76 \%$ de las personas respondieron que sí estaban dispuestas a pagar por el servicio de recolección (figura 9).

Otro factor a favor de ampliar las rutas hacia estas comunidades es el alto porcentaje de consentimiento de las personas de recibir el servicio y pagar por él. Si bien es cierto, una vez se inicie la recolección $24 \%$ de las personas no estarán dispuestas a pagar, la Municipalidad deberá realizar esfuerzos administrativos para la recuperación de dineros que generen morosidad, sin tener que llegar en últimas instancias al cobro judicial.

\section{Figura 9}

Cantidad de usuarios dispuestos a pagar por el servicio de recolección de servicios sólidos

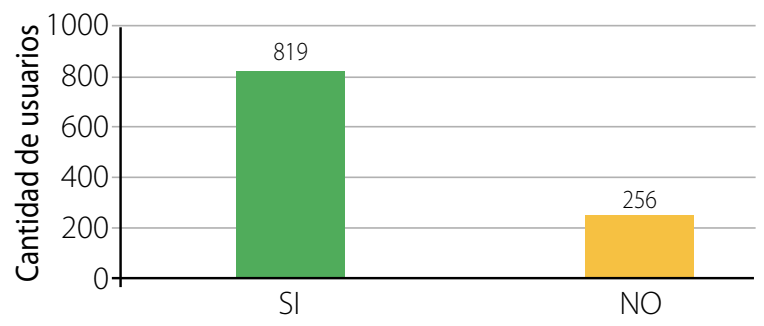

Nota. Elaboración propia con base en datos de la Municipalidad de Upala (2021).

\section{Conclusiones}

El cantón de Upala genera aproximadamente 212 toneladas por semana, actualmente se recolectan, en promedio, 70 toneladas (Municipalidad de Upala, 2019); por lo tanto, en caso de realizar una ampliación hacia estas comunidades, por lo menos una vez a la semana, se necesitaría aumentar la flota vehicular, además del personal adicional requerido y el acondicionamiento de las carreteras 
para su ingreso. Caso contrario, se podría pensar en una tercerización del servicio hacia las comunidades.

El aumento de cobertura de aproximadamente $8 \%$, contribuirá a disminuir por su parte el porcentaje de residuos sólidos quemados o enterrados, mediante lo cual se mejorará la salud ambiental de los potenciales usuarios del servicio y se disminuirá la cantidad de $\mathrm{CO}_{2}$ emitida a la atmósfera, simultáneamente, la Municipalidad mejoraría el índice de gestión municipal.

Así bien, $76 \%$ de los potenciales usuarios del servicio de recolección están de acuerdo con pagar por el servicio, por consiguiente, la Municipalidad de Upala se aseguraría que la morosidad por el 'no pago' no vaya a superar $24 \%$ que, desde luego, con una correcta gestión del cobro y con un servicio eficiente, será un porcentaje menor.

La utilización de la aplicación Input redujo tiempos en la toma de información geoespacial al igual que errores de inconsistencias o digitalización. La información al encontrarse en un almacenamiento de datos de internet, está disponible para un análisis inmediato.

La utilización de la aplicación Input aporta control en la calidad de los datos, es decir, la fecha y hora de cuándo se realizó el levantamiento y las coordenadas geográficas donde se tomaron. A su vez, permitió definir las posibles rutas de recolección donde deberá transitar el camión recolector.

La utilización de la aplicación Input también redujo la cantidad de recursos insumos necesarios para el levantamiento geoespacial, como lo fueron el recurso humano y el uso de papel, lápiz o lapicero, dispositivo GPS (Global Position Satelital).

\section{Recomendaciones}

En caso de que se decida ampliar la cobertura de recolección hacia las comunidades de Santa Cecilia, Las Milpas, Armenias, Las Brisas, Aguas Claras, Guayabal, Río Negro y La Torre, se debe implementar, previo a su inicio, un programa de comunicación dirigido a la población para asegurar que todos los públicos (comercial y residencial) conozcan el detalle de los horarios, rutas y demás especificaciones, con la meta de que la implementación sea exitosa.

La suma anual por recuperar de $\measuredangle 58120140$ por el servicio de recolección, no podrá generar pérdidas en función de los costos directos e indirectos, por lo que se deberá aplicar anualmente una actualización tarifaria del servicio de recolección.

Es necesario implementar un programa de educación para la población con el fin de que disponga los residuos a la hora indicada y tener mecanismos más rigurosos en caso de que no cumplan con las disposiciones de la municipalidad, de lo contrario, no se podrá cumplir con los objetivos de optimizar los recorridos en las rutas.

Se debe incentivar a los comercios a separar los residuos valorizables de forma que puedan ser recolectados independientemente de los que se envían a un relleno sanitario, valorando mecanismos como la premiación o la sanción (según corresponda) en caso de que se realice o no la adecuada separación de los residuos. 


\section{Referencias}

Escuela de Ciencias Geográficas (2006). Capas de información cartográfica: Plan regulador para el cantón de Upala. Universidad Nacional, Heredia, Costa Rica.

Hidrogeotecnia (2019). Estudio de optimización de las rutas de recolección de residuos sólidos ordinarios del cantón de Upala. Municipalidad de Upala, Alajuela, Costa Rica

Instituto Nacional de Estadística y Censo (2012). X Censo Nacional de Población y VI de Vivienda 2011. San José, Costa Rica.

Ley de Gestión Integral de Residuos Sólidos, Ley 8839. (2010). Asamblea Legislativa de Costa Rica. San José, Costa Rica.

Lutra Consulting (2019). Store and track changes to your geo-data. Mergin. https://public.cloudmergin.com/

Lutra Consulting (2019). Input (8.0) [Aplicación móvil]. https://play.google.com/store/apps/details?id=uk. co.lutraconsulting\&ah= GSqwibzO2n63iMICjHmMuBk89t4\&pcampaignid=MKT-Other-global-all-co-prtnr-py-PartBadge-Mar2515-1

Municipalidad de Upala (2021). Proyecto Ampliación de las Rutas de Recolección de Residuos Sólidos en los Distritos de Aguas Claras, Canalete, Bijagua y Upala. Alajuela, Costa Rica.

Pacheco Prado, D. F. (2013). Infraestructuras de datos espaciales en dispositivos móviles inteligentes. (Tesis de maestría en publicación). Universidad del Azuay, Cuenca, Ecuador.

QGIS Development Team, (2021). QGIS Geographic Information System. Open Source Geospatial Foundation Project. https://agis.org.

Soto Córdoba, S. (2019). Gestión de los Residuos Sólidos en Costa Rica. Informe Estado de la Nación en Desarrollo Humano Sostenible 2019. San José, Costa Rica. 